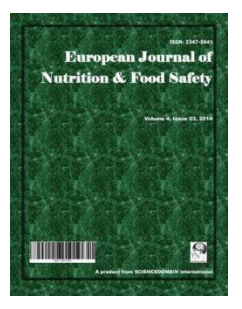

European Journal of Nutrition \& Food Safety

5(5): 985-986, 2015, Article no.EJNFS.2015.390

ISSN: 2347-5641

SCIENCEDOMAIN international

www.sciencedomain.org

\title{
Anaemia in Pregnancy: Factors Associated with Maternal Anaemia in the Dadaab Refugee Camps, Kenya (2001-2002)
}

\author{
James Smith ${ }^{1^{*}}$, Laird Ruth ${ }^{2}$, Ismail Kassim ${ }^{1}$, Allison Oman ${ }^{3}$, \\ Caroline Wilkinson ${ }^{4}$ and Andrew Seal ${ }^{1}$ \\ ${ }^{1}$ University College London, London, UK. \\ ${ }^{2}$ Nutrition Branch, Centers for Disease Control and Prevention, Atlanta, USA. \\ ${ }^{3}$ United Nations High Commissioner for Refugees, Nairobi, Kenya. \\ ${ }^{4}$ United Nations High Commissioner for Refugees, Geneva, Switzerland.
}

Article Information

DOI: 10.9734/EJNFS/2015/21199

Special Editors:

Lucie Bohac, Micronutrient Forum Secretariat, Canada.

Klaus Kraemer, Director, Sight and Life, Basel, Switzerland.

Chief Editor

Prof. Hans Verhagen, Senior Scientific Advisor 'Nutrition and Food Safety', National Institute for Public Health and the Environment (RIVM), P.O.Box 1, 3720 BA, Bilthoven, The Netherlands.

Conference Abstract

Received $5^{\text {th }}$ February 2015

Accepted $1^{\text {st }}$ March 2015

Published $14^{\text {th }}$ August 2015

\section{ABSTRACT}

Objectives: 1) Describe the socioeconomic and health status of this population of pregnant women;

2) Determine the anaemia prevalence using a series of diagnostic markers (haemoglobin, sTFR, and ZPP);

3) Explore the relationship between a variety of socio-demographic, nutritional, and disease-related factors, and anaemia.

Methods: This paper was developed using data collected during a longitudinal cohort study conducted between 2001-2002, which investigated the effectiveness of a new antenatal micronutrient supplement. A preliminary literature review identified known biological and socioeconomic risk factors associated with anaemia. $\mathrm{Chi}^{2}$, students $\mathrm{t}$-test, correlation, and logistic regression analyses explored the strength of association between exposure variables and anaemia.

Results: The prevalence of maternal anaemia in this population was $75 \%$ at $16-24$ weeks gestation. Iron deficiency was similarly widespread; $65 \%$ and $45 \%$ of women exceeded the threshold for deficiency as defined by ZPP and STFR indicators respectively. Malaria, 
schistosomiasis, and hookworm infections were not significantly associated with anaemia in this population. MUAC and BMI were strongly correlated with anaemia, as was Somali ethnicity, iron deficiency (sTFR), and living in a rural context pre-displacement.

Conclusions: The prevalence of anaemia and iron deficiency among pregnant women in Dadaab constituted a severe public health problem, as defined by the World Health Organisation. Since this study, a strategy for the control and reduction of anaemia has been developed by UNHCR and implemented in the Dadaab camps and elsewhere. However, data published more recently suggests that maternal anaemia remains a persistent problem $(74 \%, 2005 ; 69 \%, 2008)$ that requires further attention and a continued response.

(c) 2015 Smith et al.; This is an Open Access article distributed under the terms of the Creative Commons Attribution License (http://creativecommons.org/licenses/by/4.0), which permits unrestricted use, distribution, and reproduction in any medium, provided the original work is properly cited. 